\title{
Comparison of seasonal and longitudinal variation of daytime MSTID activity using GPS observation and GAIA simulations
}

\author{
Mani Sivakandan ${ }^{1,4^{*}}$ (D, Yuichi Otsuka', Priyanka Ghosh ${ }^{1,4}$, Hiroyuki Shinagawa ${ }^{2}$, Atsuki Shinbori ${ }^{1}$ \\ and Yasunobu Miyoshi ${ }^{3}$
}

\begin{abstract}
The total electron content (TEC) data derived from the GAIA (Ground-to-topside model of Atmosphere lonosphere for Aeronomy) is used to study the seasonal and longitudinal variation of occurrence of medium-scale traveling ionospheric disturbances (MSTIDs) during daytime (09:00-15:00 LT) for the year 2011 at eight locations in northern and southern hemispheres, and the results are compared with ground-based Global Positioning System (GPS)-TEC. To derive TEC variations caused by MSTIDs from the GAIA (GPS) data, we obtained detrended TEC by subtracting 2-h (1-h) running average from the TEC, and calculated standard deviation of the detrended TEC in $2 \mathrm{~h} \mathrm{(1} \mathrm{h).} \mathrm{MSTID} \mathrm{activ-}$ ity was defined as a ratio of the standard deviation to the averaged TEC. Both GAIA simulation and GPS observations data show that daytime MSTID activities in the northern and southern hemisphere (NH and SH) are higher in winter than in other seasons. From the GAIA simulation, the amplitude of the meridional wind variations, which could be representative of gravity waves (GWs), shows two peaks in winter and summer. The winter peak in the amplitude of the meridional wind variations coincides with the winter peak of the daytime MSTIDs, indicating that the high GW activity is responsible for the high MSTID activity. On the other hand, the MSTID activity does not increase in summer. This is because the GWs in the thermosphere propagate poleward in summer, and equatorward in winter, and the equatorward-propagating GWs cause large plasma density perturbations compared to the poleward-propagating GWs. Longitudinal variation of daytime MSTID activity in winter is seen in both hemispheres. The MSTID activity during winter in the NH is higher over Japan than USA, and the MSTID activity during winter in the SH is the highest in South America. In a nutshell, GAIA can successfully reproduce the seasonal and longitudinal variation of the daytime MSTIDs. This study confirms that GWs cause the daytime MSTIDs in GAIA and amplitude and propagation direction of the GWs control the noted seasonal variation. GW activities in the middle and lower atmosphere cause the longitudinal variation.
\end{abstract}

Keywords: Daytime MSTIDs, GAIA model, GPS, Gravity waves, Meridional wind, TEC

\section{Introduction}

Several investigators have studied the traveling ionospheric disturbances (TIDs) since 1940, using a variety of techniques (Beynon 1948; Bowman 1968; Chan

\footnotetext{
*Correspondence: skandanm89@gmail.com; mani.sivakandan@isee. nagoya-u.ac.jp

${ }^{1}$ Institute for Space-Earth Environmental Research, Nagoya University, Nagoya 464-8601, Japan

Full list of author information is available at the end of the article
}

and Villard 1962; Chimonas and Hines 1970; Davis and Darosa 1969; Francis 1973; Heisler 1957; Martyn 1959; Munro 1950; Pierce and Mimno 1940; Soicher 1988). The most successful interpretation of these observations is that the TIDs are corresponding to disturbances of the neutral atmosphere associated with the passage of internal atmospheric gravity waves (GWs) (Hines 1960). Then, theoretical explanation of precise nature of the causal connection between the GW and the resulting 
ionospheric disturbances was given by Hooke (1968). This theory suggests that meridional component of neutral wind oscillation due to the GWs can effectively generate the TIDs through the ion-neutral collision process. Based on the characteristics of wavelength and phase speed, TIDs are classified into two categories, viz largescale TIDs (LSTIDs) and medium-scale TIDs (MSTIDs) (Hunsucker 1982). LSTIDs have horizontal wavelength longer than $1000 \mathrm{~km}$, phase speed of 400-1000 m/s, and period ranging from $30 \mathrm{~min}$ to $3 \mathrm{~h}$. Horizontal wavelength, phase speed, and period of MSTIDs are several hundred kilometres, $250-1000 \mathrm{~m} / \mathrm{s}$, and $15 \mathrm{~min}$ to $1 \mathrm{~h}$, respectively. MSTIDs have been observed by various techniques (Amorim et al. 2011; Duly et al. 2013; Frissell et al. 2014; Kotake et al. 2007; Narayanan et al. 2014; Otsuka et al. 2013).

It is well understood that LSTIDs are mostly generated during the geomagnetic disturbed conditions (Francis 1973; Gardner and Schunk 2010, 2011; Tsugawa et al. 2003; Zhou et al. 2012). On the other hand, it has been believed that MSTIDs are generated in association with GWs originated in the lower/middle atmosphere. MSTIDs are observed both in the daytime and in the nighttime. In the northern hemisphere $(\mathrm{NH})$, during the daytime they frequently propagate towards south or southeast direction, and in the nighttime the preferential propagation direction is southwest with phase front alignment in the northwest to southeast direction. The difference in the propagation direction of MSTIDs suggests that the causative mechanism of daytime and nighttime MSTIDs is different (Kotake et al. 2006; Tsugawa et al. 2007). The nighttime MSTID is caused by the electrodynamical processes (i.e. Perkins instability associated with E- and F-region coupling processes) (Cosgrove et al. 2004; Makela and Otsuka 2012; Otsuka et al. 2007, 2009; Saito et al. 2008), and the daytime MSTID is generated by the atmospheric GWs (MacDougall et al. 2009). In this study, we are focusing on the relationship between the daytime MSTIDs and GWs. The nighttime MSTIDs, which could be generated by the electrodynamical processes, are beyond the scope of this study.

Although there are many investigations that deal with the daytime MSTIDs (e.g., Figueiredo et al. 2018; Huang et al. 2019) earlier, mechanisms which determine the seasonal variation of the daytime MSTIDs are still argued. Using 3-year TEC data at six different regions in the world, Kotake et al. (2006) studied the seasonal, longitudinal and latitudinal variations of the daytime and nighttime MSTID activities. They found that the daytime MSTID activity is higher during winter in all the six regions, and suggested that GWs could be a possible causative mechanism of the daytime MSTIDs. Park et al. (2014) showed the maximum occurrence of medium-scale GWs in winter by using the thermospheric density data obtained on the CHAMP (Challenging Minisatellite Payload) satellite. Using 1-year data of SuperDARN radar, Frissell et al. (2014) reported the local time dependency of the daytime MSTID occurrence, and argued that in addition to GWs generated in the lower atmosphere, geomagnetic activity may have some contribution to the generation of the daytime MSTIDs. Modelling studies on daytime MSTIDs could give an exclusive information of the source of generation of MSTIDs. However, such modelling studies are limited.

Recently, using a month data of a high-resolution version of GAIA model in solar minimum year of 2009, Miyoshi et al. (2018) studied the role of GWs originated in the lower atmosphere for the generation of daytime MSTIDs, and pointed out that the simulated MSTIDs have similar characteristics to those obtained with GPSTEC observations. Their results suggest that GAIA can successfully reproduce the daytime MSTIDs. In the present study, we investigate the seasonal and longitudinal variations of the daytime MSTID activity in both northern and southern hemispheres for the year 2011 by using the high-resolution GAIA model simulations, and the obtained results are compared with the GPS-TEC observations. Moreover, we also made an attempt to understand the possible causative mechanism for the seasonal and longitudinal variations of the daytime MSTIDs.

\section{Data set and method of analysis}

The method carried out to estimate the MSTID activity from the observation and model simulations are detailed in this section.

\section{GPS-TEC data}

TEC is calculated from pseudorange and carrier phase data obtained with dual-frequency GPS receivers installed all over the world. To study the seasonal and longitudinal variations of MSTID activity, we used GPS data for the whole year of 2011 at eight different regions consisting of four locations in each hemisphere viz., Western United States of America $\left(35^{\circ} \mathrm{N}, 115^{\circ} \mathrm{W}\right)$ hereafter WUSA, Eastern United States of America $\left(45^{\circ} \mathrm{N}\right.$, $\left.68^{\circ} \mathrm{W}\right)$ hereafter EUSA, Europe $\left(45^{\circ} \mathrm{N}, 09^{\circ} \mathrm{E}\right)$ and Japan $\left(35^{\circ} \mathrm{N}, 140^{\circ} \mathrm{E}\right)$ in the $\mathrm{NH}$ and South America $\left(35^{\circ} \mathrm{S}, 73^{\circ}\right.$ $\mathrm{W})$, South Africa $\left(25^{\circ} \mathrm{S}, 27^{\circ} \mathrm{E}\right)$, Australia $\left(25^{\circ} \mathrm{S}, 134^{\circ} \mathrm{E}\right)$ and New Zealand $\left(44^{\circ} \mathrm{S}, 169^{\circ} \mathrm{E}\right)$ in the $\mathrm{SH}$. The locations of the GPS receivers used in the present study are shown in Fig. 1. Two receivers are used in Europe, South Africa, and Australia, respectively, three receivers are used in WUSA, EUSA, South America, and New Zealand, respectively, and six receivers are used in Japan.

Perturbation components of TEC are derived by subtracting the 1-h running average from the time sequence 


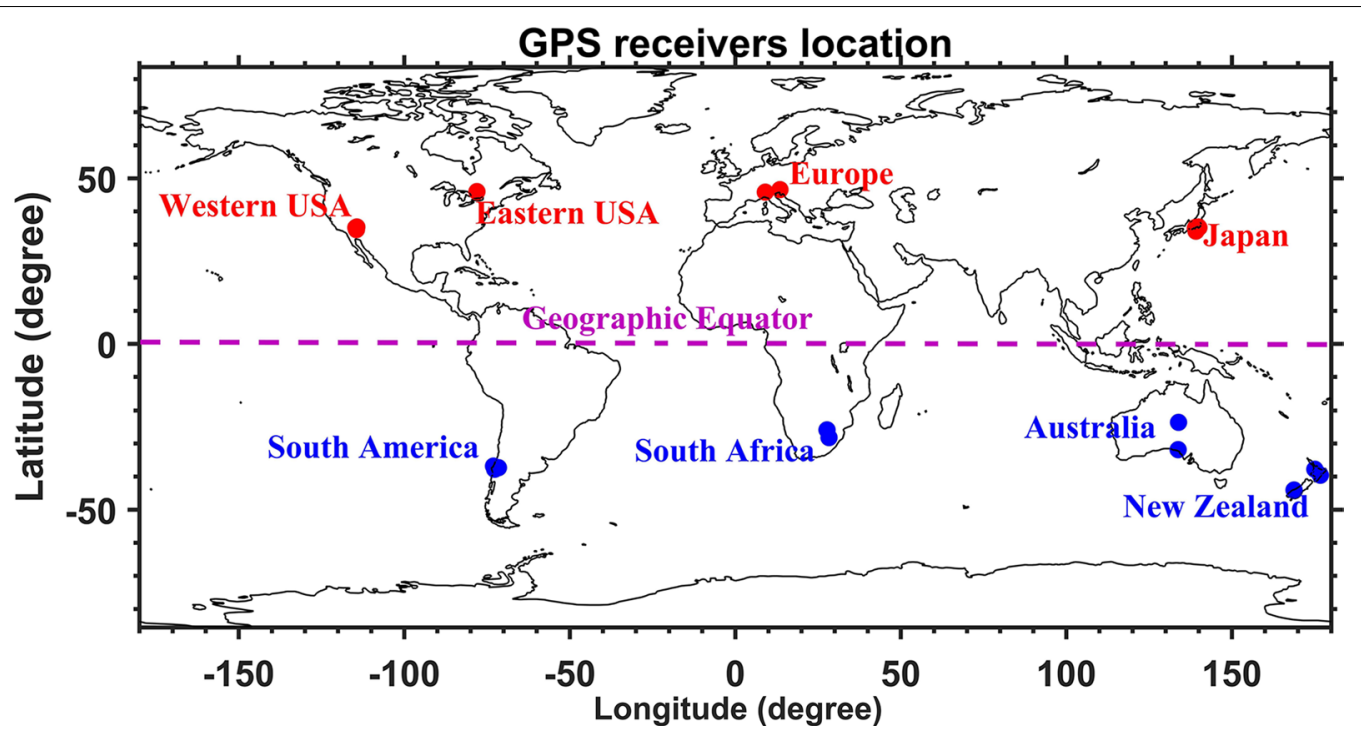

Fig. 1 Location of the GPS receivers used in the present study

of TEC for each satellite receiver pair. Then, the perturbation component of slant TEC is converted into the vertical TEC by multiplying the slant factor (Saito et al. 1998). Perturbations obtained from this method are believed to be caused by MSTIDs (Kotake et al. 2006). To study the MSTID activity statistically, we calculated the standard deviation of the perturbation TEC within $1 \mathrm{~h}$ for each satellite receiver path every hour. The MSTID activity is defined as the ratio between the standard deviation of the perturbation and 1-h average absolute TEC. The MSTID activity is statistically studied by Kotake et al. (2006). Their results show that seasonal and local time variations of the MSTID activity are consistent with those of MSTID occurrence rate obtained from visual inspection of two-dimensional maps of TEC perturbations (Kotake et al. 2007; Otsuka et al. 2011) and 630-nm nighttime airglow observations (e.g., Shiokawa et al. 2003). The absolute TEC is derived based on the method reported by Otsuka et al. (2002). In this study, both perturbation and absolute TEC with satellite elevation angle larger than $55^{\circ}$ are used in order to reduce effects of cycle slips. The MSTID activities obtained from different satellites and receivers are averaged for each region. Monthly averages of MSTID activities are calculated to study seasonal and longitudinal variations of MSTID activity.

\section{An overview of the GAIA model}

The Ground-to-topside of Atmosphere and Ionosphere for Aeronomy (GAIA) model is an atmosphere-ionosphere coupled model that covers whole atmospheric region from the ground surface to the upper thermosphere/ionosphere without any artificial boundaries between the lower and upper regions. It is an integration of three models, viz. a General Circulation Model (GCM) of the neutral atmosphere, an ionosphere model, and an electrodynamics model. A detailed description of GAIA can be found in the following references (Jin et al. 2011; Miyoshi et al. 2011, 2012; Shinagawa et al. 2017). In the present study, we have used a high-resolution version of GAIA model with horizontal and vertical resolution of about $1^{\circ}$ in longitude by about $1^{\circ}$ in latitude and 0.2 scale height, respectively. This means that the model can resolve atmospheric waves with horizontal wavelength larger than $375 \mathrm{~km}$ (Miyoshi et al. 2018). This model includes a full set of physical processes applicable for the whole atmospheric region. Schemes for moist convection, a hydrological cycle, boundary layers, and radiation as well as the effect of mountains and land-sea contrasts are also included. In the thermosphere, the model incorporates the interaction processes between the plasma and the neutral species, such as ion drag, Joule heating, and auroral precipitation heating. GAIA uses tilted dipole magnetic field. The output data with temporal resolution of $10 \mathrm{~min}$ are analysed in this study.

Atmospheric waves with horizontal scales less than $2000 \mathrm{~km}$ in the troposphere and lower stratosphere are spontaneously generated in the model. This means that GWs are generated via meteorological processes, such as moist convection, surface topography, and jet/front instability. Furthermore, the model can simulate the upward propagation of these GWs from the troposphere to the middle and upper atmospheres. For the ionospheric part, the dynamics and chemical processes of the major ion species $\left(\mathrm{O}^{+}, \mathrm{O}_{2}{ }^{+}, \mathrm{N}_{2}{ }^{+}\right.$, and $\left.\mathrm{NO}^{+}\right)$are taken into account 
(Shinagawa 2009). The coupling processes between the plasma and the neutrals are also included. In this simulation, day-to-day variations in the F10.7 $\mathrm{cm}$ flux are taken into account, and geomagnetically quiet condition is assumed. In the present study, GAIA-simulated TEC data are used to derive the MSTID activity by the same method of the GPS observation but perturbation components of TEC are derived by subtracting the 2 -h running average from the sequence of vertical TEC and standard deviation of the perturbation is calculated in 2-h. Note that TEC in GAIA is an integration of the plasma density in altitude below $3170 \mathrm{~km}$. The MSTID activity is defined as the ratio between the standard deviation of the perturbation and 2-h averaged absolute TEC. Then, monthly averages of the MSTID activity are calculated to study the seasonal variation. To identify the possible causative mechanism of seasonal variations of MSTID activity, meridional wind and electron density are used to investigate GW activity and effect of GW on the plasma density perturbations in the ionosphere.

\section{Results}

In the present study, we validate the GAIA-simulated daytime MSTID activities with GPS observations by investigating seasonal and longitudinal variations of the MSTID activity at different locations at middle latitudes. Furthermore, the meridional wind perturbations simulated by GAIA are used to argue the possible causative mechanisms for the seasonal and longitudinal variations of daytime MSTID.

\section{Seasonal variation of daytime MSTID activity: northern hemisphere}

Figure 2 shows seasonal and local time variations of (top) GPS-observed MSTID activity, and (middle and bottom)
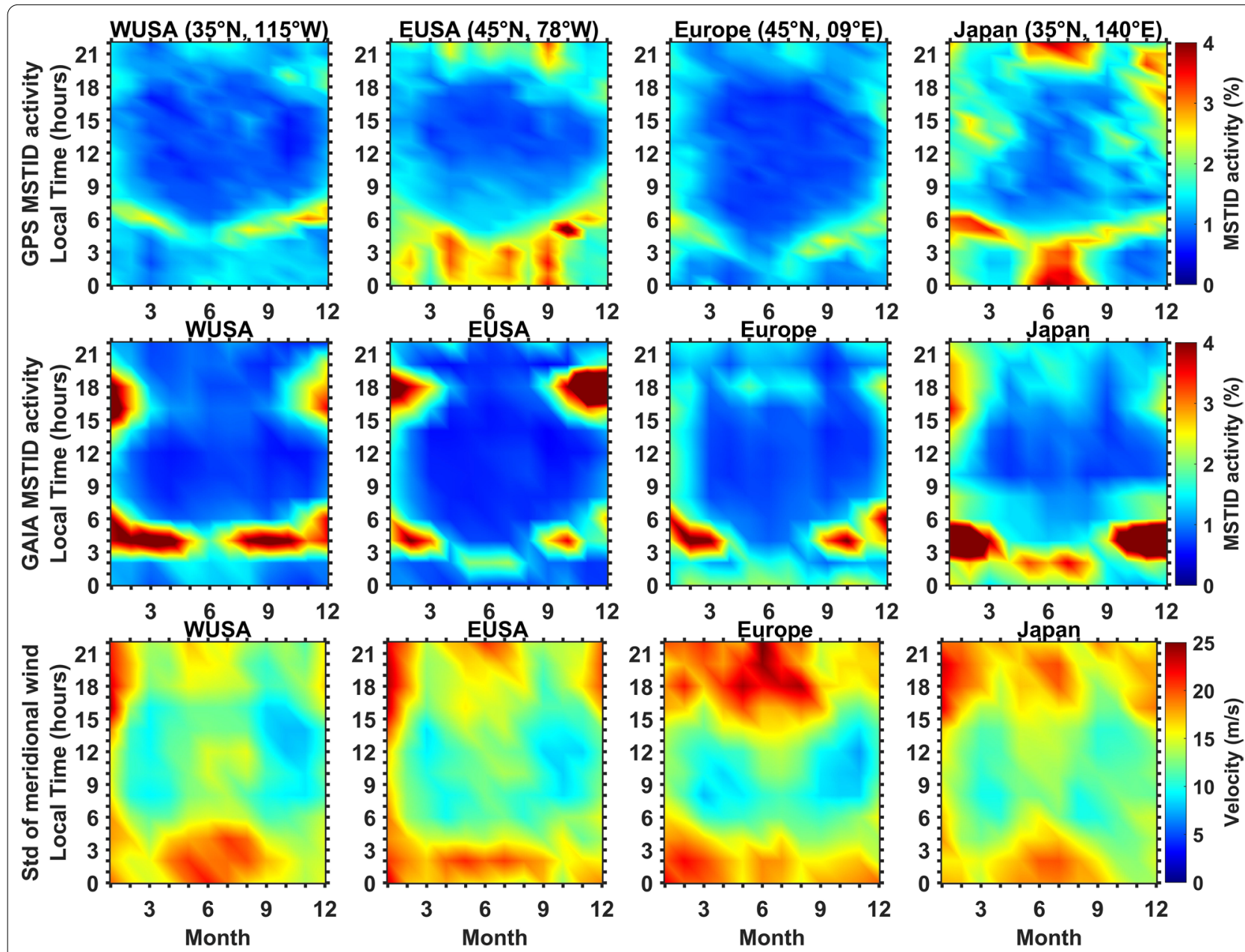

Fig. 2 Local time and seasonal variations of (top and middle) MSITD activities obtained from GPS observation and GAIA model simulation, and (bottom) standard deviation of meridional wind variation at an altitude of $300 \mathrm{~km}$ in GAIA model at four different locations in the northern hemisphere, viz. Western USA, Eastern USA, Europe and Japan 
MSTID activity and standard deviation of meridional wind perturbations obtained from the GAIA simulation, respectively, at different regions, viz. WUSA, EUSA, Europe and Japan in the NH for the year 2011. In each panel, the horizontal axes and vertical axes represent the month and local time (LT), respectively. It should be noted that an enhancement of the MSTID activity at sunrise (03:00-07:00 LT) and sunset (17:00-19:00 LT) is not the signature of MSTID, but the effect of the rapid increase (decrease) of TEC. Therefore, these enhancements around sunrise and sunset are not considered for the discussion. From the top and middle panels, it is clear that MSTID activities obtained from both GPS observations and GAIA simulation are smaller during the daytime than during the nighttime. Similarly, the standard deviation of meridional wind variation is smaller during the daytime than the nighttime. Both GPS observations and GAIA simulation show that the daytime MSTID activity at all of the four locations is the highest in winter, and that the maximum activity during the daytime occurs at different local time for the different locations. For example, the daytime MSTID activity is the highest around 09:00-15:00 LT in winter (particularly, January and February) over the WUSA and EUSA in GAIA and 09:00-12:00 LT in GPS observations. The standard deviation of meridional wind variation at these locations are high around 09:00-15:00 LT during winter. In Japan and Europe, the daytime MSTID activity and wind variation are larger in the afternoon. In the European sector, the daytime MSTID activity is less in the noon (12:00-13:00 LT) and higher before noon and afternoon in both observation and simulation.

In order to investigate the seasonal variation of MSTID activity, we have averaged the MSTID activity and standard deviation of wind variation over daytime (09:00-15:00 LT) for all the four locations (Fig. 3). In Fig. 3, the left vertical axis denotes the MSTID activity, and the right vertical axis indicates the standard deviation of zonal and meridional wind variation. The MSTID activity from the observation and GAIA simulation is shown by the red and black curves, respectively. Error bar with the observed MSTID activity represents its standard deviation. The standard deviation of zonal and meridional wind variation is shown by the blue curves. In all the four locations, the maximum daytime MSTIDs occur during the winter months (December, January and February) in both observation and model simulations. A secondary peak appears during the summer months (June, July and August) in WUSA (in both GPS observation and GAIA simulation) and European in GPS observation. The secondary peak is not seen in the EUSA and Japanese sectors. This implies that GAIA can successfully

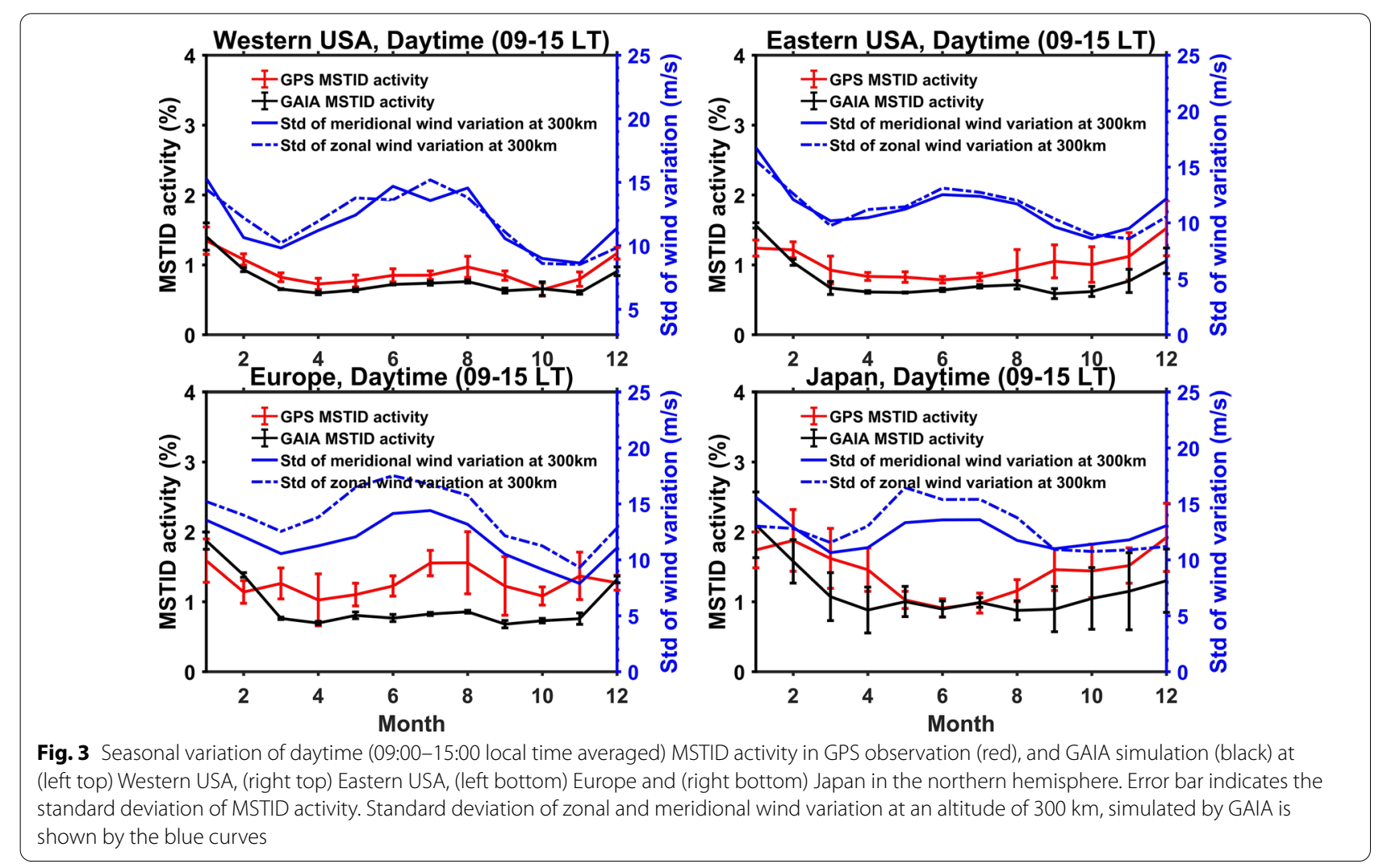


reproduce the seasonal variation of the daytime MSTID activity. Furthermore, the standard deviation of zonal and meridional wind variation simulated by GAIA also shows a primary peak in winter and a secondary peak in summer in the EUSA and Japanese sectors. Over WUSA, the primary peak is noticed in January (a winter month) and the secondary peak is observed during summer as well as in December (a winter month). In contrast, over the Europe, the primary peak is observed in summer and the secondary peak is observed in winter. Amplitude of the zonal and meridional wind variations which could be caused by GWs in the thermosphere show semi-annual variations with maxima in solstices and minima in equinox whereas that daytime MSTID activity is the largest in winter. The wind variation peak during winter suggests that neutral atmospheric GW activity may be the potential source for the generation of MSTIDs in GAIA simulation (further details are given in the discussion section).
Most of the month's observed MSTID activity is higher than the GAIA-simulated one in all the four locations.

\section{Seasonal variation of daytime MSTID activity: southern hemisphere}

Figure 4 shows the seasonal and local time variations of daytime MSTID activity obtained from GPS observation and GAIA simulation and standard deviation of meridional wind variation over the $\mathrm{SH}$ for the year 2011. The format of this figure is the same as that of Fig. 2, but for South America, South Africa, Australia and New Zealand in SH. MSTID activity and standard deviation of wind variations are smaller during the daytime than those during the nighttime at all the four locations, which is similar to the NH. Both GPS observation and GAIA simulation show that the daytime MSTID activity in SH is primarily high during winter months (May, June and July). The standard deviation of meridional wind variation during daytime is also high during the winter at all the four

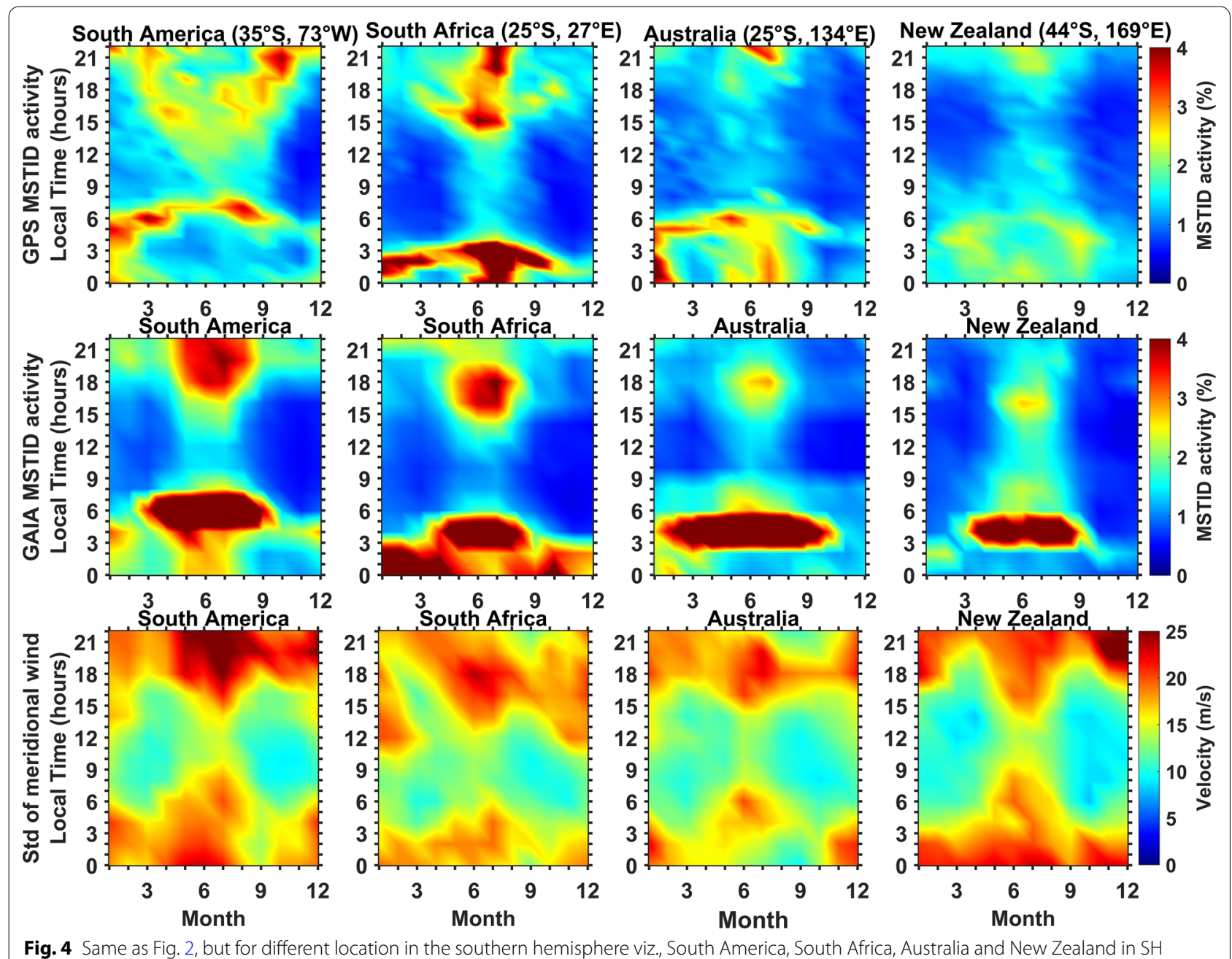

Fig. 4 Same as Fig. 2, but for different location in the southern hemisphere viz., South America, South Africa, Australia and New Zealand in SH 
locations. The standard deviation of meridional wind variation during daytime is higher over South America and New Zealand than South Africa and Australia.

To understand the seasonal variation of the daytime MSTIDs, we have averaged the MSTID activity over daytime between 09:00 and 15:00 LT in the same way as that for $\mathrm{NH}$. Figure 5 shows seasonal variation of daytime-averaged MSTID activity in GPS observation (red), and GAIA simulation (black) at the four locations in the southern hemisphere. The standard deviation of the observed MSTID activity is represented as error bar. Maximum of the daytime MSTID activity appears during winter (May, June and July) in both observation (red) and model simulation (blue). The seasonal variation of the daytime MSTID activity obtained from GPS observations is well reproduced in the GAIA model whereas the daytime MSTID activity in South America in the GAIA simulation is smaller than that in the GPS observations. On the other hand, the standard deviation of zonal and meridional wind variation from the GAIA simulation (blue dashed curve) shows two peaks; one is in winter and the other is in summer whereas standard deviation of zonal (blue curve with dot) wind variation at Australia does not show clear seasonal variation. Note that from Figs. 3, 5, it is clear that most of the locations the seasonal variation of MSTID activity is larger than the standard deviation. Therefore, the seasonal difference is significant enough. The standard deviation of both zonal and meridional wind variation show similar seasonal variation with comparable amplitude except for South America. At South America, standard deviation of meridional wind show a peak in winter, but such a peak is not discernible for zonal wind variations. Seasonal variation of the meridional wind variations resembles that of the MSTID activity rather than zonal wind variations, suggesting that the meridional component of the wind variation control the MSTIDs. The further discussion focuses on the meridional wind variations. Peak value in the standard deviation of meridional wind variation is comparable between winter and summer at South America $(\sim 15.5 \mathrm{~m} / \mathrm{s}$ in summer and $\sim 15.3 \mathrm{~m} / \mathrm{s}$ in winter $)$ and Australia $(\sim 13.9 \mathrm{~m} / \mathrm{s}$ in summer and $\sim 13.5 \mathrm{~m} / \mathrm{s}$ in winter). On the other hand, at South Africa, meridional wind variation in summer is higher $(\sim 15.5 \mathrm{~m} / \mathrm{s})$ than winter $(\sim 13.9 \mathrm{~m} / \mathrm{s})$. The winter maximum of standard deviation of wind coincides with maximum of daytime MSTID activity, suggesting that GWs can be a possible source of daytime MSTID. However, the double peak of wind variation in both winter and summer raises a question. Why is the MSTID activity small in summer although the amplitude of the meridional wind variations is high? The plausible reason is investigated in the discussion section.

\section{Longitudinal variation of daytime MSTID activity}

We studied the longitudinal variation of daytime MSTID activity by comparing the peak amplitudes of daytime
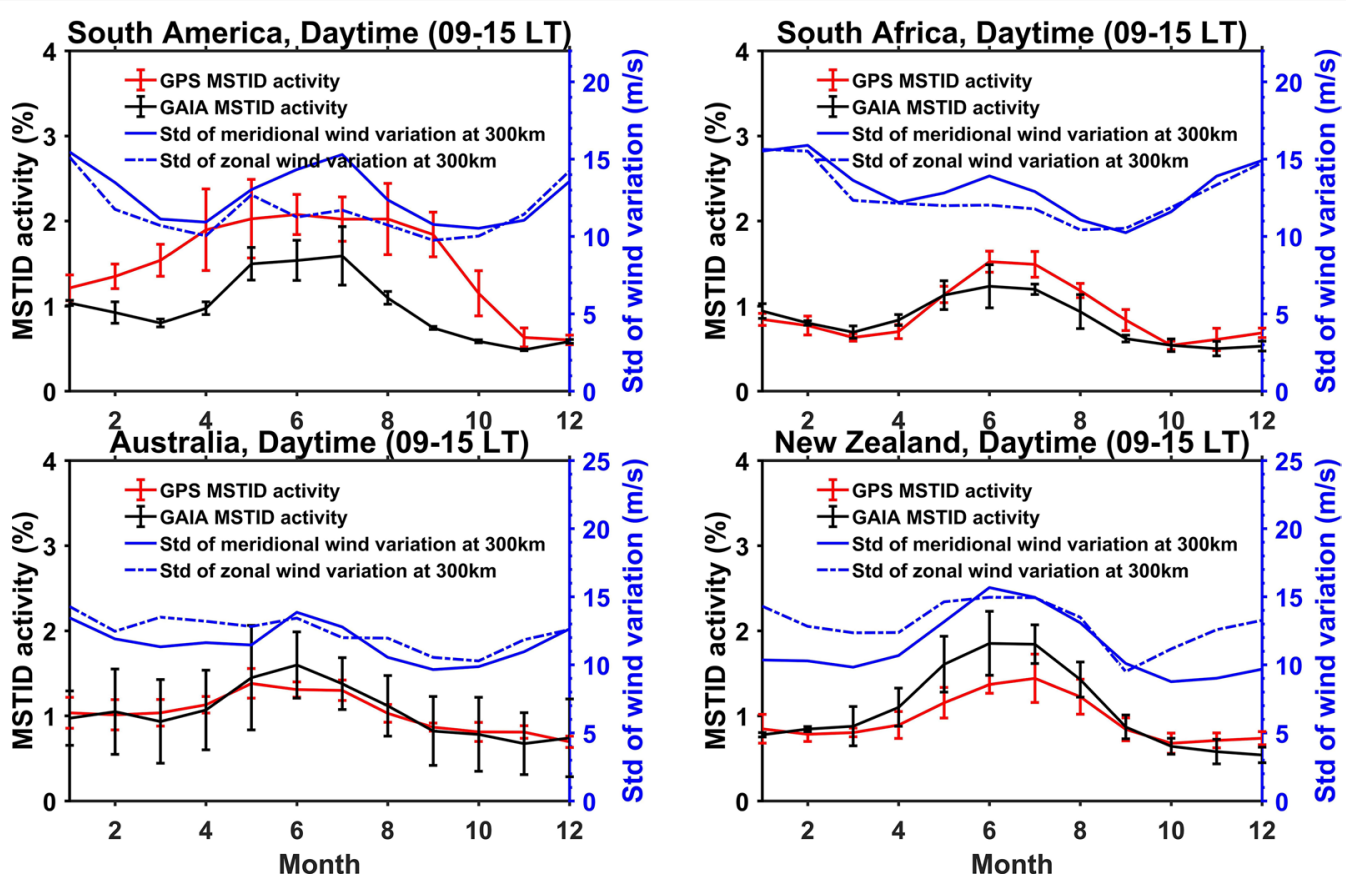

Fig. 5 Same as Fig. 3, but different locations in the southern hemisphere, viz. South America, South Africa, Australia and New Zealand in SH 
MSTID activity in all the four locations in GAIA and GPS observation for the $\mathrm{NH}$ and $\mathrm{SH}$ separately. Please note here that, the peak amplitude is the maximum MSTID activity observed in a particular season for each location. Longitudinal variation of daytime MSTID activity in (left) $\mathrm{NH}$ and (right) $\mathrm{SH}$ is shown in Fig. 6. In NH, the peak daytime MSTID activity is higher in Japan (1.9\% and $2.0 \%)$ than USA (1.3\% and $1.4 \%$ ) in both observation and model simulation. GPS observation shows maximum MSTID activity in South America (2.0\%) and minimum

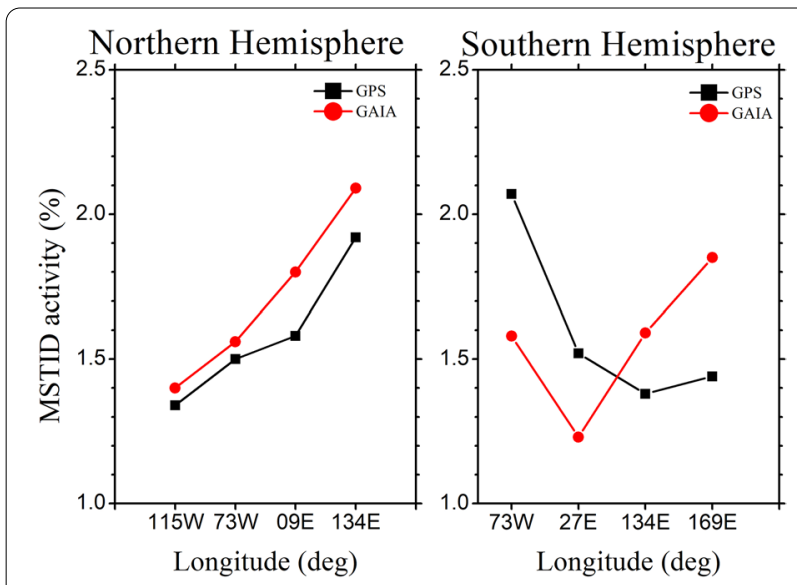

Fig. 6 Comparison of longitudinal variation of daytime MSTID activity during the maximum occurrence period (i.e. January in northern hemisphere and July in southern hemisphere) in GPS observation (black lines) and GAIA simulation (red lines) in (left) $\mathrm{NH}$ (right) SH. Horizontal axis denotes longitude, and vertical axis represents the MSTID activity in Australia (1.4\%) in SH. Longitudinal variation of the daytime MSTID activity is reversed from that in NH. However, the GAIA simulation shows a maximum in New Zealand (1.8\%), a minimum in Africa (1.2\%), and a secondary maximum in South America (1.6\%). Over all, in $\mathrm{NH}$, GAIA can successfully reproduce the longitudinal variation of the daytime MSTID activity, but in $\mathrm{SH}$ it shows some discrepancy, especially over the South American sector.

To investigate the causative mechanism of longitudinal variation of the daytime MSTID activity in GAIA model, we compared the MSTID activity with the standard deviation of meridional wind variation during winter as a function of longitude. Figure 7 shows the comparison of the MSTID activity with standard deviation of meridional wind variation in winter in (left) $\mathrm{NH}$ and (right) $\mathrm{SH}$. In $\mathrm{NH}$, the wind variation shows similar tendency (i.e. higher in Japanese sector than USA) with MSTID activity at all the three locations except Europe in December. In $\mathrm{SH}$, on the other hand, the daytime MSTID activity and standard deviation of wind variation show similar tendency, which is a decrease from the South American sector $\left(73^{\circ} \mathrm{W}\right)$ to the South African sector $\left(27^{\circ} \mathrm{E}\right)$ and an increase towards the New Zealand sector $\left(169^{\circ} \mathrm{E}\right)$.

\section{Discussion}

Using TEC acquired from the dual-frequency GPS receiver data, we derived the MSTID activity at different locations in both northern and southern hemispheres (four locations in each hemisphere) for the year 2011, and studied the seasonal and longitudinal variations of the daytime MSTID activity and compared the seasonal and longitudinal

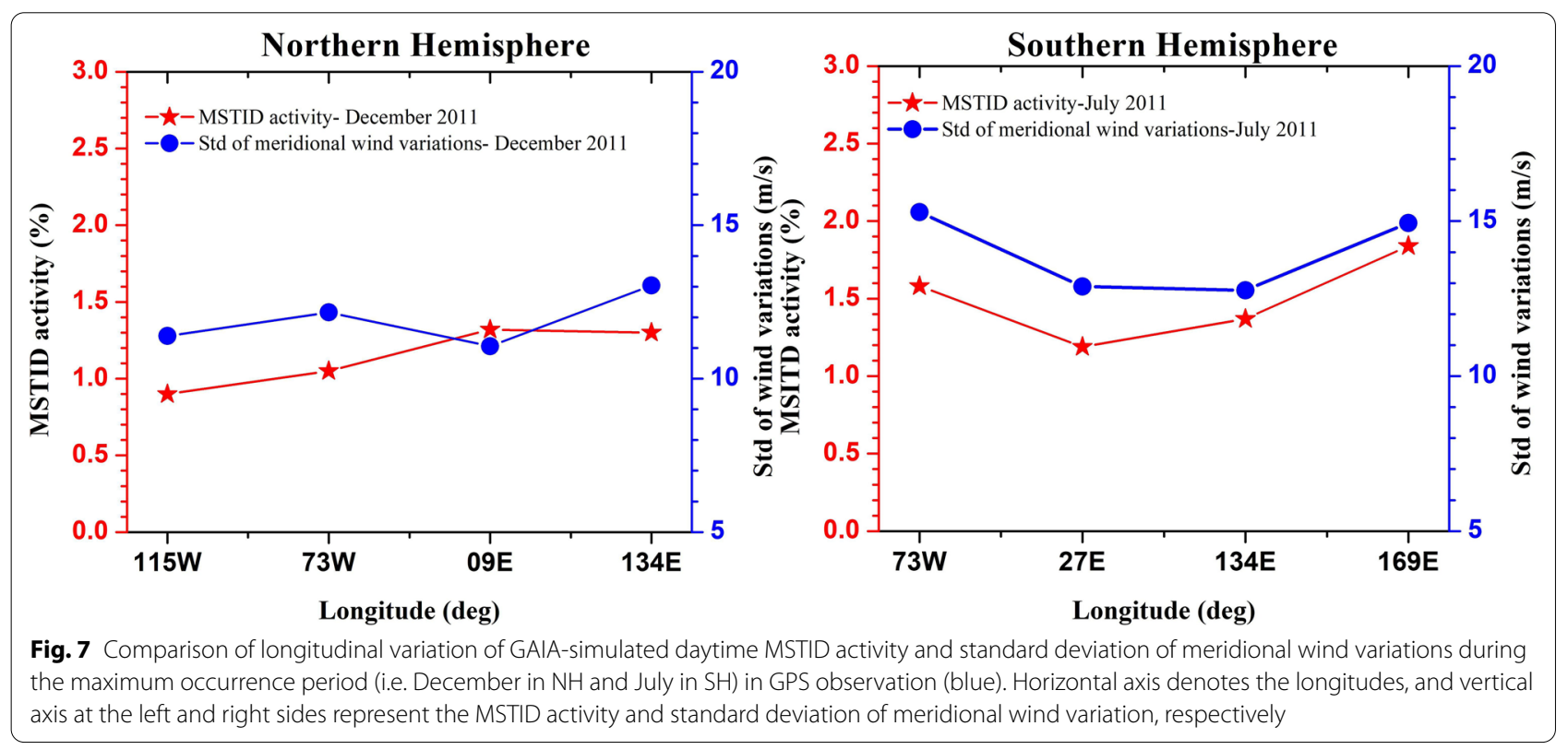


variations of the GAIA-simulated MSTID activity with GPS-observed MSTID activity for each location of the GPS receivers. Additionally, using the standard deviation of meridional wind variations simulated by GAIA, we also investigate the possible causative mechanism of seasonal and longitudinal variations of daytime MSTID activity.

There are many studies of MSTID during daytime. Most of these studies are carried out in the $\mathrm{NH}$ (Ishida et al. 2008; Ding et al. 2011; Frissell et al. 2014, 2016; Onishi et al. 2009) and few studies over the SH (e.g., Figueiredo et al. 2018; Huang et al. 2019; Vadas et al. 2019). Moreover, most of them are case studies. In literature, few studies reported the seasonal variation of daytime MSTIDs using GPS-TEC or satellite in situ observation. They suggest that irrespective of geographical longitudes the daytime MSTID occurrence is more in winter than summer in both $\mathrm{NH}$ and $\mathrm{SH}$ (Kotake et al. 2006; Park et al. 2014). In the present investigation, the GPS-TEC observations and GAIA simulation shows that the daytime MSTID in both NH and SH is higher in winter than in other seasons. This result is consistent with the previous studies. We have found that GAIA model can successfully reproduce the seasonal variation of daytime MSTIDs (Figs. 2, 3, 4, 5). Furthermore, the standard deviation of zonal and meridional wind variations, which is representative of neutral atmospheric perturbations in the GAIA model also displays seasonal variation of a primary peak in winter at most of the locations and a secondary peak in summer in both $\mathrm{NH}$ and $\mathrm{SH}$.

Seasonal variation of GWs in the thermosphere shows semi-annual variations with maxima in solstices and minima in equinox whereas that daytime MSTID activity is the largest in winter. However, the primary peak of the standard deviation of meridional wind variations has good agreement with the seasonal variation of daytime MSTID activity. This suggests that GW cause the daytime MSTIDs, and that the seasonal variation of the daytime MSTID activity is controlled by that of GW activity, as suggested by earlier reports (Bowman 1968; Chimonas and Hines 1970; Hunsucker 1982).

In the F-region, ions move only along the geomagnetic field line (B) through the ion-neutral collisions whereas the ion motion across $B$ is restricted because the ion gyro-frequency is much higher than the ion-neutral collision frequency. (Hooke 1968) showed the electron density perturbation $\left(\frac{N_{e}}{N_{e}}\right)$ caused by the GW in the ionosphere, as follows:

$$
\frac{N_{e}^{\prime}}{N_{e}}=i \omega^{-1} u_{\|}\left[\frac{1}{N_{e}} \frac{\partial N_{e}}{\partial z} \sin I-i k_{\|}\right],
$$

where $\omega$ is the angular frequency of the $\mathrm{GW}, u_{\|}$and $k_{\|}$are the neutral wind perturbation velocity and wave number of the GW parallel to the ambient magnetic field, respectively, and $I$ is the magnetic dip angle. GW in the neutral atmosphere causes field aligned ion motion with velocity equal to $u_{\|}$through ion-neutral collision. The first term of the right-hand side in Eq. (1) indicate the electron density perturbations caused by the vertical displacement of the $F$ layer, At the bottom side of the F layer, where $\frac{\partial N_{e}}{\partial z}$ is positive, $\frac{N_{e}}{N_{e}}$ decreases (increases) when the ion moves upward (downward) (Nelson 1968). The second terms of the right-hand side in Eq. (1) indicates the convergence and divergence of ions along the geomagnetic field line. It should be noted that the neutral particle motion caused by GWs is incompressible, but that the ion is compressible because the ion motion is restricted to the direction parallel to the geomagnetic field.

As per Eq. (1), the electron density perturbation $\left(\frac{N_{e}^{\prime}}{N_{e}}\right)$ is proportional to neutral wind perturbation velocity parallel to $\mathrm{B}\left(u_{\|}\right)$, that is, $\left|\frac{N_{e}^{\prime}}{N_{e}}\right| \alpha\left|u_{\|}\right|$. This suggests that GW with large amplitude could cause larger electron density perturbation than that with smaller amplitudes (Hooke 1968). Present GAIA simulations show that amplitude of meridional wind perturbation is larger in winter. This suggests that the GWs induced large electron density perturbations in winter. As a result, MSTID activity also becomes high in winter.

As shown in the result section, the seasonal variation of the standard deviation of meridional wind variation has two peaks, viz. a winter primary peak and summer secondary peak. The winter peak of the meridional wind variation coincides with the winter peak of the MSTID activity. At South Africa, the wind variation in summer is higher than in winter, but the MSTID activity is smaller in summer than winter. In order to understand the plausible reason for this inconsistent feature, we have looked into the seasonal variation of standard deviation of daytime monthly mean meridional wind variations (top panel) and electron density variations (middle panel) as well as the monthly mean TEC and MSTID activity (bottom panel) over the South African sector $\left(25^{\circ} \mathrm{S}, 27^{\circ} \mathrm{E}\right)$, as shown in Fig. 8. The meridional wind and electron density variations are estimated by subtracting the 2 -h running average of meridional wind and electron density, respectively. Using the daytime (07:00-17:00 LT) meridional wind and electron density variations standard deviation of wind and electron density variation for every $2 \mathrm{~h}$ are calculated for each day. Followed by the daytime (09:00-15:00 LT) mean and monthly mean of wind and electron density standard deviation are calculated. The altitude profile of monthly mean of daytime meridional wind and electron density variations are shown in Fig. 8a, b, respectively. From these figures, it is found that magnitude of the wind variation around $200-300 \mathrm{~km}$ altitudes is comparable in summer (in particular January 

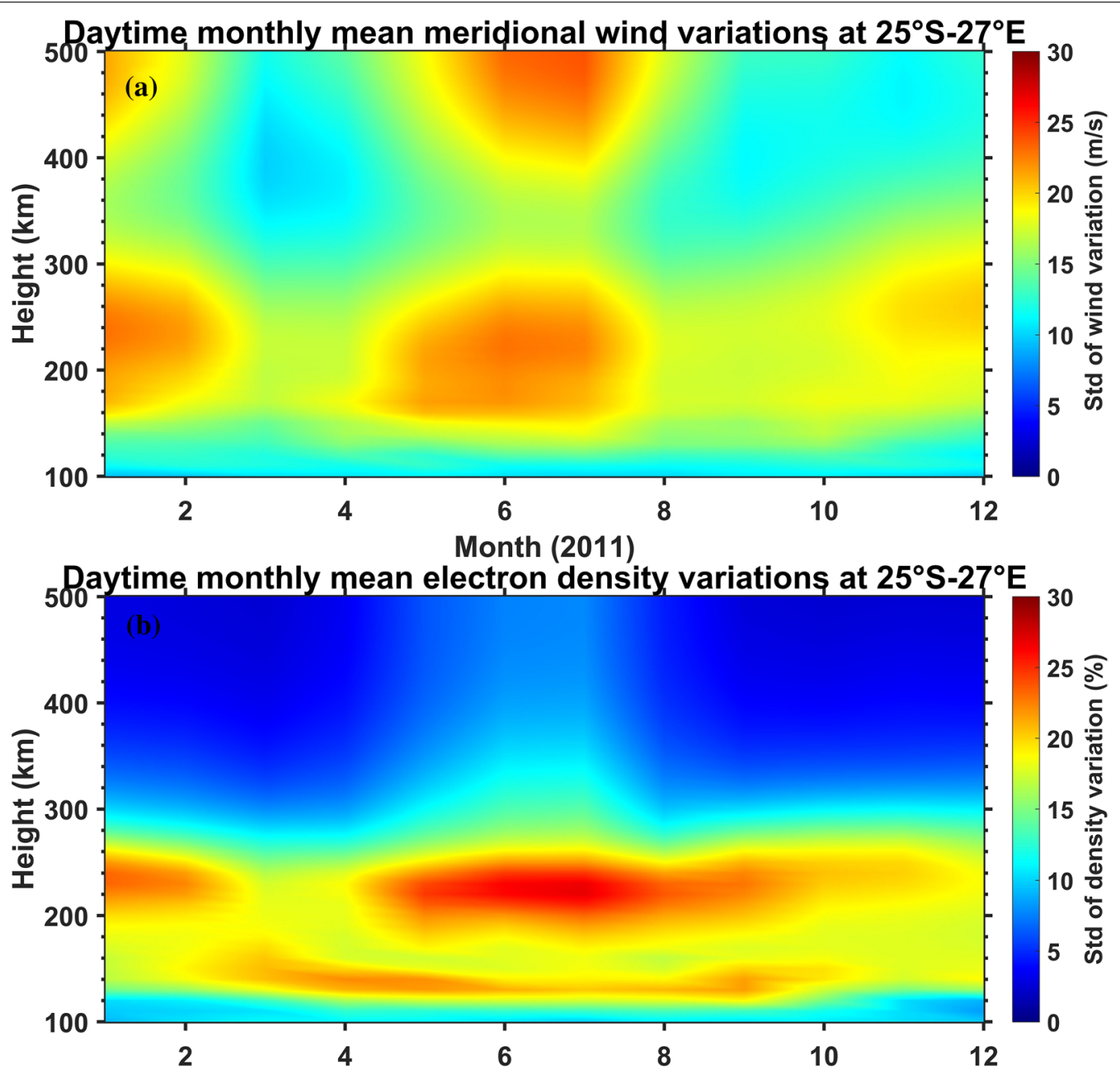

Month (2011)

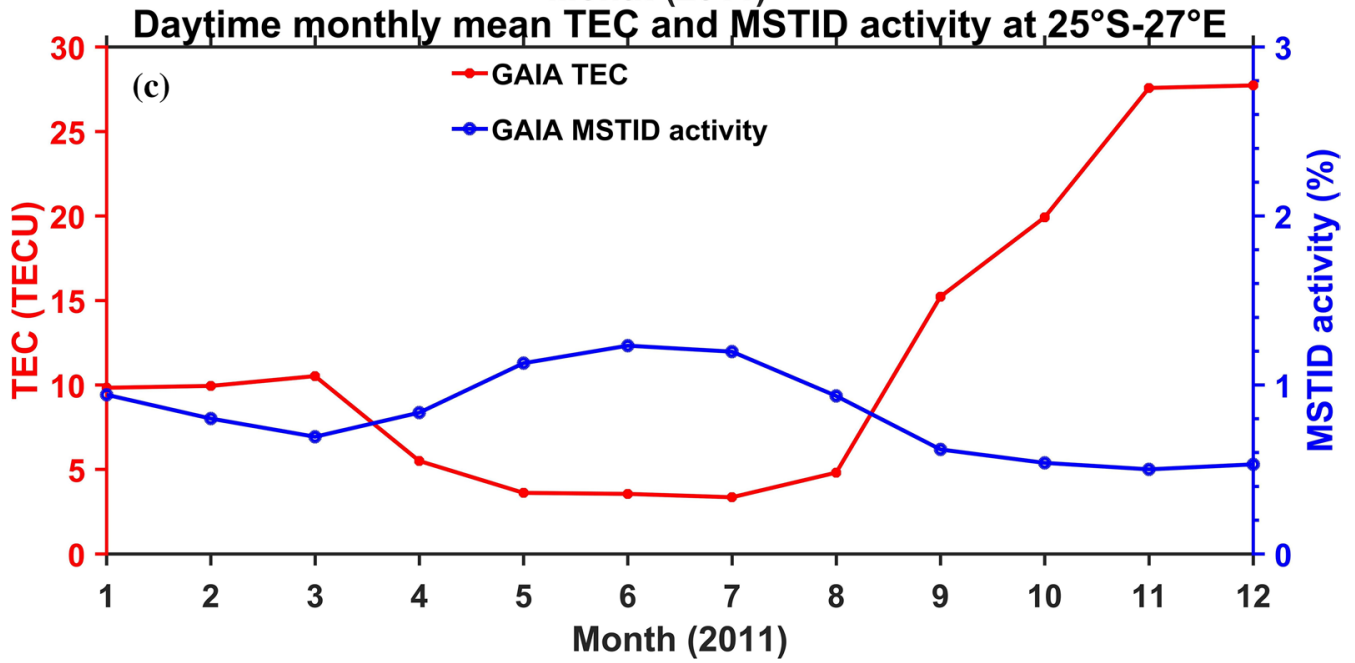

Fig. 8 Altitude and seasonal variations of the daytime (09:00-15:00 LT) monthly mean standard deviation of (top) meridional wind variations and (middle) electron density variations over the South African $\left(25^{\circ} \mathrm{S}, 27^{\circ} \mathrm{E}\right)$ for the year 2011 . Bottom panel show daytime time monthly mean TEC and MSTID activity over South Africa in the same period 
and February) and winter (May-June-July) although on December, magnitude of the wind variation is less than that of January and February. On the other hand, the electron density variation around 200-300 km altitudes is higher in winter than summer (Fig. 8b). Consequently, as shown in Fig. 8, the MSTID activity is higher in winter. It also raises a following question. Although the amplitude of wind variation is comparable between summer and winter, why is the electron density variation higher during winter than summer?

To clarify the above question, we investigate effects of the propagation direction of the GWs on MSTID activity. In GAIA simulation, Miyoshi et al. (2018) have shown that the GWs propagate equatorward in winter hemisphere and poleward in summer hemisphere. An important point to be noted here is that on average the trans-hemispheric thermosphere wind blows from summer hemisphere to winter hemisphere, which means the meridional wind flow equatorward (poleward) direction in summer (winter) hemisphere. Therefore, the GWs propagate windward. This feature can be explained as below. The linear dispersion relation for high frequency GWs is expressed as

$$
m^{2}=\frac{N^{2}}{(c-u)^{2}}
$$

where $m$ is the vertical wavenumber, $N$ is the Brunt Väisälä frequency, $c$ is the phase speed and $u$ is the background wind in the direction of wave propagation. When GWs propagate in the same direction as the background wind, $(c-u)^{2}$ becomes small, resulting in increase in $m$ and decrease in the vertical wavelength of GWs. The GWs with small vertical wavelength could likely dissipate due to the viscosity. When GWs propagate in the opposite direction to the background wind, the vertical wavelength becomes large. This condition could allow the GWs to propagate upward for a long distance with little dissipation. In this study, using the GAIA simulations, we investigate meridional wind and plasma density variations on typical days in winter (07 June 2011) and summer (08 January 2011) over South Africa in SH. Left panels of Fig. 9 show the altitude-latitude cross section (20-30 $\mathrm{S}$ in latitudes) of (top) meridional wind variation and (bottom) electron density variation at 13:42 LT on 08 January 2011. In these figures, magnetic field (taken from GAIA) line with a constant dip angle of $-45^{\circ}$ is also shown as a black dotted line. The right panels show the latitudinal average of standard deviation of (top) meridional wind variation and (bottom) electron density variation at 13:42 LT. It is to be noted here is that in the current case, the standard deviation of wind and electron density variations are calculated over the period of 12:42-14:42 LT (the time when the MSTID is observed), and the obtained standard deviations are averaged over the latitudes from $20^{\circ} \mathrm{S}$ to $30^{\circ} \mathrm{S}$. From the left panels, it is found that the phase fronts are elongated from upward-poleward to downward-equatorward, indicating poleward propagation of GW. In the right panels, the maximum amplitude of standard deviation of wind variation $(\sim 25 \mathrm{~m} / \mathrm{s})$ and electron density variation $(\sim 16 \%)$ are noted around an altitude of $250 \mathrm{~km}$. The format of Fig. 10 is same as that of Fig. 9, but for 07 June 2011 standard deviation of wind and electron density variations are calculated over the period of 14:42-16:42 LT, when daytime MSTID in winter is seen. In left panels of Fig. 10, the phase front of the perturbations is elongated from upward-equatorward to poleward-downward, indicating the equatorward propagation of GW. From the right panels, it is found that maximum amplitude of (top) mediational wind and (bottom) electron density variations, which appear around an altitude of $230 \mathrm{~km}$, are $15.6 \mathrm{~m} / \mathrm{s}$ and $19 \%$, respectively. Comparing the amplitude of the meridional wind and electron density variations between summer and winter, it is found that the amplitude of the meridional wind perturbation is larger in summer than winter, but that the electron density variation is larger in winter than summer. This result indicates that propagation direction of GWs affect magnitude of the electron density variations resulting in MSTID.

Due to GWs, the neutral particles oscillate in the direction parallel to the phase front of GWs in the horizontal distance and altitude cross section. Therefore, for the poleward-propagating GWs, the neutral particles oscillate in the direction from upward-poleward to downward- equatorward. This direction is nearly perpendicular to the geomagnetic field lines. Instead, for the equatorward-propagating GWs, the oscillation of neutral particle is from upward-equatorward to poleward-downward. The direction is nearly parallel to the geomagnetic field lines. As mentioned earlier, the ions in the F-region move only along the geomagnetic field line through the ion-neutral collisions. The ion perturbation velocity induced by GWs is equal to $u_{\|}$, which is the neutral wind velocity projected to the geomagnetic field line. The ion perturbation velocity is larger for the equatorward-propagating GWs than that for the poleward-propagating GWs because the neutral wind velocity perturbation parallel to the geomagnetic field line is larger for equatorward-propagating GWs than the poleward-propagating GWs. Therefore, the present result suggests that propagation direction of the $\mathrm{GW}$ is responsible for the fact that the amplitude of electron density perturbation and thus, daytime MSTIDs are larger in winter than summer. Consequently, the 

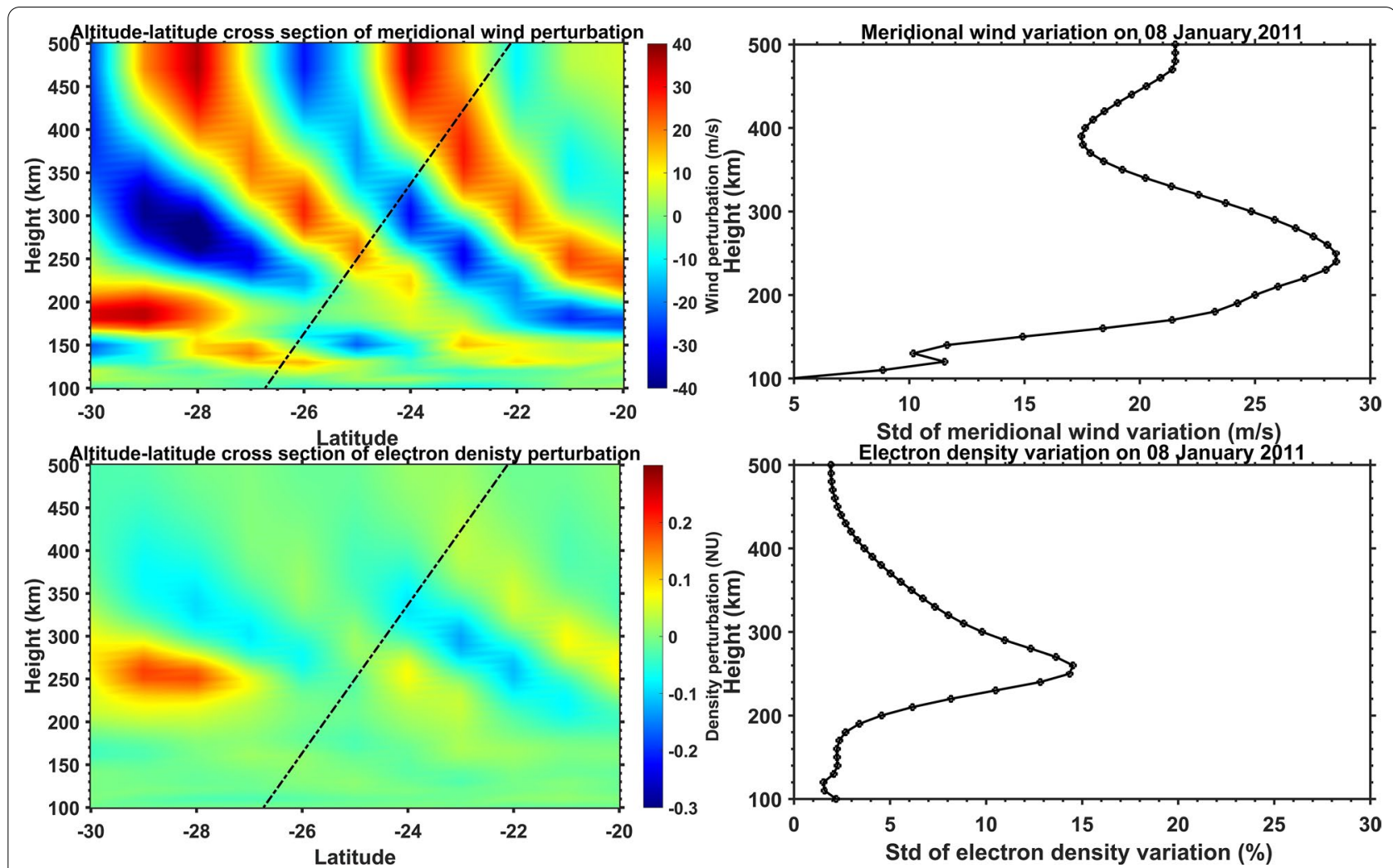

Fig. 9 Results of GAIA simulation at South Africa at 13:42 LT on 8 January, 2011: (Left) altitude-latitude cross section of (top) meridional wind and (bottom) electron density perturbations and altitude-latitude variation of a magnetic field (taken from GAIA model) line with constant dip angle $\left(\sim 45^{\circ}\right)$ at $250 \mathrm{~km}$ is also shown (black dotted lines) in these figures. (Right) Altitude profiles of standard deviation of (top) meridional wind and (bottom) electron density variations. The standard deviations are averaged over 20-30 $\mathrm{S}$ latitudes

seasonal variation of standard deviation of meridional wind variation shows two peaks in winter and summer, whereas the MSTID activity shows a peak in winter.

In the latitude-altitude cross section of wind variation, shown in the top left panels of Figs. 9, 10, the phase front of GW is not connected below an altitude of approximately $120 \mathrm{~km}$, suggesting that the GWs causing the daytime MSTIDs do not propagate directly from the lower atmosphere. This suggests that the GWs causing the daytime MSTIDs in the ionosphere are secondary GWs which might be generated around the mesosphere and lower thermosphere region at altitudes of $90-120 \mathrm{~km}$ (Miyoshi et al. 2018; Vadas et al. 2019). When the zonal winds in the stratosphere and/or mesosphere are strong, upward propagating GWs are likely to dissipate because the most of GWs reach their critical level and the vertical wavelength of GWs becomes short. The secondary GWs are excited when a dissipation of GWs occurs. Therefore, the secondary GWs are likely excited in summer and winter when the winds are strong (Miyoshi et al. 2015, 2018). The secondary GWs can propagate in all directions except the direction perpendicular to the body force direction (Vadas and Liu 2009) so that from dissipation of zonally propagating GWs by the zonal winds, the secondary GWs having the meridional component of the propagation can be excited. Upward propagation of these secondary GWs into the thermosphere could be responsible for the seasonal variation of GWs activity in the thermosphere with two peaks in winter and summer.

The GPS-TEC observations show that notable longitudinal variation of daytime MSTID in both hemispheres; the daytime MSTID activity in winter in NH is higher over Japan than USA, and the MSTID activity in winter in $\mathrm{SH}$ is the highest in South America. This feature is consistent with an earlier report by Kotake et al. (2006), who have shown the MSTID activity obtained from the GPS-TEC data in the world in the three years (1998, 2000, and 2001). Using the 10-year data of neutral density measured on the CHAMP satellite, Park et al. (2014) have studied the climatology of daytime medium-scale GWs in the thermosphere. Their findings show that during local winter at mid-latitudes (in general), thermospheric GW activity shows much stronger longitudinal variation in the $\mathrm{SH}$ than $\mathrm{NH}$, and that 

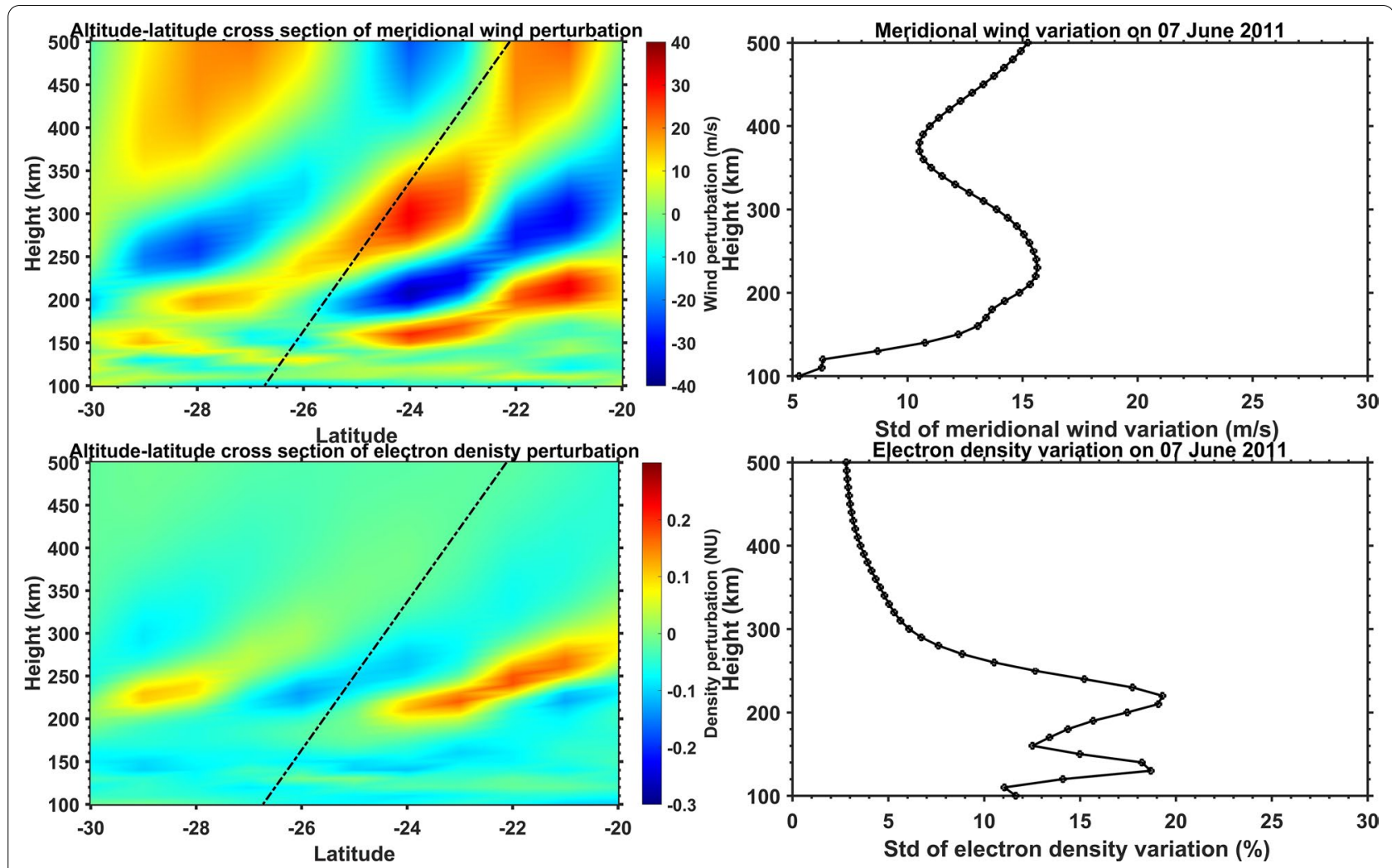

Fig. 10 Same as Fig. 9, but for a typical day (07 June 2018) at 15:42 LT in SH winter

during June solstice, GW activity near Andes and Antarctic peninsula is higher than any other locations. Trinh et al. (2018), who have analysed thermospheric neutral density obtained on the satellite missions of the GOCE and CHAMP, have shown that GW activities in winter in $\mathrm{NH}$ are higher at EUSA and Japan, and that GW activities in winter in $\mathrm{SH}$ are higher over the South American sector. These seasonal and longitudinal variations of the daytime medium-scale GWs are consistent with those of the MSTID activity shown in the present study. Especially, both MSTID activity and GWs in the thermosphere is high in winter over South America. Jiang et al. $(2003,2004)$ have shown that GW variances at an altitude of $\sim 38 \mathrm{~km}$ in the stratosphere, which could be mountaingenerated GWs are larger in winter in both $\mathrm{NH}$ and $\mathrm{SH}$. Mountain waves break in the middle atmosphere and could generate secondary and tertiary GWs, as discussed above. Vadas et al. (2019) analysed traveling atmospheric disturbances (TADs) over the southern Andes observed by GOCE satellite on 5 July 2010. They found that nearly all of the GWs had intrinsic horizontal phase velocity of $245-630 \mathrm{~m} / \mathrm{s}$, they argued that GWs with such large horizontal phase velocity could not come from below $\sim 80 \mathrm{~km}$ in altitude because acoustic velocity in the lower atmosphere is approximately $300 \mathrm{~m} / \mathrm{s}$. Thus, they suggested that the TAD hotspots observed over the Andes/Antarctic peninsula in winter results from the generation of secondary and tertiary GWs (i.e. multi-step processes). These results suggest that high daytime MSTIDs activity over South America, shown in GPS observations, could be caused by high activity of mountain GWs launched from Andes Mountains.

As shown in Fig. 6, GAIA well reproduces the longitudinal variation of the daytime MSTID activity, especially in the NH, where the daytime MSTID activity is the highest over Japan and the lowest over WUSA. Amplitude of meridional wind variations simulated by GAIA also show similar longitudinal variations (Fig. 7), suggesting that the longitudinal variation of the GW activity in the thermosphere is responsible for the longitudinal variation of the daytime MSTID activity. In the SH, both daytime MSTID and amplitude of the wind variations simulated by GAIA are larger at South America and New Zealand (Fig. 7). However, they do not show a distinct enhancement at South America, unlike the MSTID activity obtained from the GPS-TEC observations.

Note that there is difference in the dip angle derived from GAIA (tilted dipole magnetic field) and actual dip angle. The dip angle taken from GAIA is compared with that taken from International Geomagnetic Reference 
Field (IGRF) 13 model (http://www.geomag.bgs.ac.uk/ data_service/models_compass/igrf_calc.html). The difference of the dip angles at the eight locations where the MSTID activity is investigated in this study is found to be less than $4^{\circ}$ except South Africa and South America, where the dip angle difference is $\sim 18^{\circ}$ and $\sim 7^{\circ}$, respectively. Consequently, the dip angle discrepancy in GAIA could not cause significant discrepancy in MSTID activity except South Africa and South America. At $\left(25^{\circ} \mathrm{S}\right.$, $27^{\circ} \mathrm{E}$ ) in South Africa, the dip angles taken from GAIA and IGRF are $-45^{\circ}$ and $-63^{\circ}$, respectively. According to Eq. (1), the electron density perturbation depends on the dip angle as $u_{\|}$, sinI, and $k_{\|}$depend on the dip angle. Thus, difference in the dip angle causes difference in the electron density perturbation amplitude. As an example, we consider an equatorward-propagating $\mathrm{GW}$ with horizontal wavelength of $666 \mathrm{~km}$, vertical wavelength of $170 \mathrm{~km}$, and amplitude of the neutral wind perturbation of $15.6 \mathrm{~m} / \mathrm{s}$. These parameters are taken from GW simulated by GAIA for the case over South Africa in summer. The estimated electron density perturbation caused by the above GW at dip angles of $-45^{\circ}$ and $-63^{\circ}$ is $15.5 \%$ and $11.9 \%$ to the background plasma density, respectively. Consequently, the dip angle discrepancy may cause approximately $30 \%$ overestimation of MSTID activity in GAIA. This difference is comparable to the difference between MSTID activities obtained from GAIA and observation at South Africa in winter, but the MSTID activity in GAIA is smaller than the observed MSTID activity. Likewise, at south America $\left(35^{\circ} \mathrm{S}, 73^{\circ}\right.$ $\mathrm{W})$, the dip angles taken from GAIA and IGRF are $-43^{\circ}$ and $-36^{\circ}$, respectively. For the above mentioned wave parameters, the dip angle discrepancy of $7^{\circ}$ at South America is estimated to cause 6\% difference of MSTID activities. However, the difference between GAIA and observed MSTID activity is 33\% (see Fig. 5), that is 27\% larger than the dip angle discrepancy estimation of GAIA and IGRF. Therefore, the dip angle discrepancy in GAIA may not be a major reason of difference between MSTID activities obtained from GAIA and observation.

Using high-resolution general circulation model, Miyoshi et al. (2014) studied the latitude-longitude variations of GW energy in the thermosphere at June and December solstices. They have shown that at middle latitudes between 25 and $45^{\circ} \mathrm{S}$ in $\mathrm{SH}, \mathrm{GW}$ activities are stronger over the New Zealand sector and South American sector than over the South African sector. This longitudinal variation of GW activity is consistent with that of amplitude in the meridional wind variations simulated in GAIA. As shown in Fig. 7, both MSTID activity and amplitude of the wind variations simulated by GAIA show the similar longitudinal variations, indicating that the daytime MSTIDs activity depend on GW activity in the GAIA simulations. Absence of the daytime MSTID activity enhancement as South America, in GAIA simulations, may be under estimation of the GW activities induced by the mountain waves launched from the Andes.

\section{Summary and conclusions}

We have statistically investigated TEC variations observed by GPS receivers for the whole year 2011 at eight locations (Western and Eastern United States of America, Europe, and Japan in the NH and South America, South Africa, Australia, and New Zealand in the SH) in order to reveal seasonal, and longitudinal variations of MSTIDs activity during daytime. Seasonal and longitudinal variations of the daytime MSTIDs simulated by the high-resolution GAIA are compared with those obtained from the GPS observations. Moreover, an attempt is made to investigate the possible causative mechanism of seasonal and longitudinal variation of daytime MSTID activity. The major findings are as follows:

a. GAIA simulation shows the amplitude of the zonal wind variation is comparable to that of the meridional wind variation and both zonal and meridional wind variations display similar seasonal variation in the thermosphere, i.e. two peaks in summer and winter. Note that zonal wind variations cannot be known from MSTID because the ion moves only along the magnetic field line.

b. The winter peak in amplitude of the meridional wind variations coincides with the winter peak in the daytime MSTID activity. This is because GWs with large amplitude are responsible for MSTIDs in winter.

c. Although amplitude of the meridional wind variations is large in summer, the MSTID activity does not increase in summer. At South Africa, the amplitude of meridional wind variations is larger in summer than winter, whereas the MSTID activity is smaller in summer than winter. The GAIA simulation shows that GWs propagate toward equator in winter and poleward in summer. The ion perturbation velocity is larger for the equatorward-propagating GWs than that for the poleward-propagating GWs because the neutral wind velocity perturbation parallel to the geomagnetic field line is larger for equatorwardpropagating GWs than the poleward-propagating GWs. Therefore, the propagation direction of the GW is responsible for the largest MSTID activity in winter.

d. The GPS-TEC observations show that notable longitudinal variation of daytime MSTID activity in winter in both hemispheres; the daytime MSTID activity in winter in $\mathrm{NH}$ is higher over Japan than USA, and 
the MSTID activity in winter in $\mathrm{SH}$ is the highest in South America. From the comparison with previous works regarding global distribution of GW activities in middle and lower atmosphere, mountain waves launched from the Andes Mountains could increase GW activities resulting in the high daytime MSTID activities over South America.

As a conclusion, GAIA can successfully reproduce the seasonal and longitudinal variation of the daytime MSTID activity. The propagation direction of GWs causes the seasonal variation. The global distribution of GW activities in the middle and lower atmosphere cause the longitudinal variation.

\begin{abstract}
Abbreviations
TEC: Total electron content; GAIA: Ground-to-topside model of Atmosphere Ionosphere for Aeronomy; IGRF: International geomagnetic reference field; GPS: Global positioning system; TIDs: Traveling ionospheric disturbances; TADs: Traveling atmospheric disturbances; MSTIDs: Medium-scale traveling ionospheric disturbances; LSTIDs: Large-scale traveling ionospheric disturbances; GWs: Gravity waves; SuperDARN: Super dual auroral radar network; CHAMP: Challenging minisatellite payload; GOCE: Gravity field and steady-state ocean circulation explorer; $\mathrm{NH}$ : Northern hemisphere; SH: Southern hemisphere; WUSA: Western United States of America; EUSA: Eastern United States of America; GCM: Global circulation model; LT: Local time.
\end{abstract}

\section{Acknowledgments}

Simulations and data analyses in this work were performed using Hitachi SR16000/M1 and the NICT Science Cloud System, Japan. M.S. acknowledges the ISEE, Nagoya University, and PSTEP and PWING project team for offering the post-doctoral fellowship to carry out the present research work.

\section{Authors' contributions}

MS carried out the major part of data analysis and wrote the manuscript. YO conceived the idea, involved in elucidation of data analysis and organization of the paper. PG was engaged in the data interpretation. HS, AS and YM participated in reviewing the paper and HS is responsible person for GAIA model simulations. All authors read and approved the final manuscript.

\section{Funding}

Open Access funding enabled and organized by Projekt DEAL. The present study was supported by the Japan Society for the Promotion of Science, KAKENHI (15H05815, 16H06286), Project for Solar-Terrestrial Environment Prediction (PSTEP) and Study of dynamical variation of particles and waves in the inner magnetosphere using ground-based network observations (PWING).

\section{Availability of data and materials}

To access the GAIA simulation data, contact H. Jin (jin@nict.go.jp). To access GPS-TEC data, contact Y. Otsuka (otsuka@isee.nagoya-u.ac.jp).

\section{Ethics approval and consent to participate}

Not applicable.

\section{Consent for publication}

Not applicable.

\section{Competing interests}

The authors declare that they have no competing interests.

\section{Author details}

${ }^{1}$ Institute for Space-Earth Environmental Research, Nagoya University, Nagoya 464-8601, Japan. ${ }^{2}$ National Institute of Information and Communications Technology, Koganei, Tokyo 184-8795, Japan. ${ }^{3}$ Earth and Planetary Sciences, Kyushu University, Fukuoka 819-0395, Japan. ${ }^{4}$ Present Address: Leibniz
Institute of Atmospheric Physics at the University of Rostock, Kühlungsborn, Germany.

Received: 7 September 2020 Accepted: 23 January 2021

Published online: 04 February 2021

\section{References}

Amorim DCM, Pimenta AA, Bittencourt JA, Fagundes PR (2011) Long-term study of medium-scale traveling ionospheric disturbances using Ol 630 $\mathrm{nm}$ all-sky imaging and ionosonde over Brazilian low latitudes. J Geophys Res Sp Phys 116:1-7. https://doi.org/10.1029/2010JA016090

Beynon WJ (1948) Short period changes in the $F$ region of the ionosphere. Nature 162:886-887

Bowman GG (1968) Movements of ionospheric irregularities and gravity waves. J Atmos Terr Phys 30:721-734

Chan KL, Villard OG (1962) Observation of large-scale traveling ionospheric disturbances by spaced-path high-frequency instantaneous-frequency measurements. J Geophys Res 67(3):973-988. https://doi.org/10.1029/ JZ067i003p00973

Chimonas G, Hines CO (1970) Atmospheric gravity waves launched by auroral currents. Planet Space Sci 18:565-582. https://doi.org/10.1016/00320633(70)90132-7

Cosgrove RB, Tsunoda RT, Fukao S, Yamamoto M (2004) Coupling of the Perkins instability and the sporadic E layer instability derived from physical arguments. J Geophys Res Sp Phys 109:1-11. https://doi. org/10.1029/2003JA010295

Davis MJ, Darosa AV (1969) Traveling ionospheric disturbances originating in the auroral oval during polar substorms. J Geophys Res 74:5721-5735. https://doi.org/10.1029/ja074i024p05721

Ding F, Wan W, Xu G et al (2011) Climatology of medium-scale traveling ionospheric disturbances observed by a GPS network in central China. J Geophys Res Sp Phys 116:1-11. https://doi.org/10.1029/2011JA016545

Duly TM, Chapagain NP, Makela JJ (2013) Climatology of nighttime mediumscale traveling ionospheric disturbances (MSTIDs) in the Central Pacific and South American sectors. Ann Geophys 31:2229-2237. https://doi. org/10.5194/angeo-31-2229-2013

Figueiredo CAOB, Takahashi H, Wrasse CM et al (2018) Medium-scale traveling ionospheric disturbances observed by detrended total electron content maps over Brazil. J Geophys Res Sp Phys 123:2215-2227. https://doi. org/10.1002/2017JA025021

Francis SH (1973) Acoustic-gravity modes and large-scale traveling ionospheric disturbances of a realistic, dissipative atmosphere. J Geophys Res 78:2278-2301. https://doi.org/10.1029/ja078i013p02278

Frissell NA, Baker JBH, Ruohoniemi JM et al (2014) Climatology of mediumscale traveling ionospheric disturbances observed by the midlatitude Blackstone SuperDARN radar. J Geophys Res Sp Phys 119:7679-7697. https://doi.org/10.1002/2014JA019870

Frissell NA, Baker JBH, Ruohoniemi JM et al (2016) Sources and characteristics of medium-scale traveling ionospheric disturbances observed by highfrequency radars in the North American sector. J Geophys Res A Sp Phys 121:3722-3739. https://doi.org/10.1002/2015JA022168

Gardner LC, Schunk RW (2011) Large-scale gravity wave characteristics simulated with a high-resolution global thermosphere-ionosphere model. J Geophys Res Sp Phys 116:1-14. https://doi.org/10.1029/2010JA015629

Gardner LC, Schunk RW (2010) Generation of traveling atmospheric disturbances during pulsating geomagnetic storms. J Geophys Res Sp Phys. https://doi.org/10.1029/2009JA015129

Heisler LH (1957) Anomalies in the ionosonde records due to traveling ionospheric disturbances. Aust J Phys 11:79-90

Hines CO (1960) Internal atmospheric gravity waves at ionospheric heights. Can J Phys 38:1441-1481. https://doi.org/10.1139/p60-150

Hooke WH (1968) lonospheric irregularities produced by internal atmospheric gravity waves. Planet Space Sci 17:749-765. https://doi. org/10.1016/0032-0633(69)90195-0

Huang F, Otsuka Y, Lei J et al (2019) Daytime periodic wave-like structures in the ionosphere observed at low latitudes over the Asian-Australian sector using total electron content from Beidou geostationary satellites. J Geophys Res Sp Phys 124:2312-2322. https://doi.org/10.1029/2018JA026443 
Hunsucker RD (1982) Atmospheric gravity waves generated in the high latitude ionosphere: a review. Rev Geophys 20:293-315. https://doi. org/10.1029/RG020i002p00293

Ishida T, Hosokawa K, Shibata T et al (2008) SuperDARN observations of daytime MSTIDs in the auroral and mid-latitudes: possibility of long-distance propagation. Geophys Res Lett 35:1-6. https://doi.org/10.1029/2008G L034623

Jiang JH, Wu DL, Eckermann SD, Ma J (2003) Mountain waves in the middle atmosphere: microwave limb sounder observations and analyses. Adv Space Res 32(5):801-806

Jiang JH, Eckermann SD, Wu DL, Ma J (2004) A search for mountain waves in MLS stratospheric limb radiances from the winter Northern Hemisphere: data analysis and global mountain wave modeling. J Geophys Res 109:D03107. https://doi.org/10.1029/2003JD003974

Jin H, Miyoshi Y, Fujiwara H et al (2011) Vertical connection from the tropospheric activities to the ionospheric longitudinal structure simulated by a new Earth's whole atmosphere-ionosphere coupled model. J Geophys Res Sp Phys 116:1-9. https://doi.org/10.1029/2010JA015925

Kotake N, Otsuka Y, Ogawa T et al (2007) Statistical study of medium-scale traveling ionospheric disturbances observed with the GPS networks in Southern California. Earth Planets Sp 59:95-102. https://doi.org/10.1186/ BF03352681

Kotake N, Otsuka Y, Tsugawa T et al (2006) Climatological study of GPS total electron content variations caused by medium-scale traveling ionospheric disturbances. J Geophys Res 111:1-8. https://doi. org/10.1029/2005JA011418

MacDougall J, Abdu MA, Batista I et al (2009) On the production of traveling ionospheric disturbances by atmospheric gravity waves. J Atmos SolarTerrestrial Phys 71:2013-2016. https://doi.org/10.1016/j.jastp.2009.09.006

Makela JJ, Otsuka Y (2012) Overview of nighttime ionospheric instabilities at low- and mid-latitudes: coupling aspects resulting in structuring at the mesoscale. Space Sci Rev 168:419-440. https://doi.org/10.1007/s1121 4-011-9816-6

Martyn DF (1959) Large-scale movements of ionization in the ionosphere. J Geophys Res 64:2178-2179

Miyoshi Y, Fujiwara $\mathrm{H}$, Jin $\mathrm{H}$ et al (2011) Model study on the formation of the equatorial mass density anomaly in the thermosphere. J Geophys Res Sp Phys 116:1-11. https://doi.org/10.1029/2010JA016315

Miyoshi Y, Fujiwara $\mathrm{H}$, Jin $\mathrm{H}$ et al (2012) Numerical simulation of the equatorial wind jet in the thermosphere. J Geophys Res Sp Phys 117:1-10. https:// doi.org/10.1029/2011JA017373

Miyoshi Y, Fujiwara H, Jin H, Shinagawa H (2015) Impacts of sudden stratospheric warming on general circulation of the thermosphere. J Geophys Res Sp Phys 120:10897-10912. https://doi.org/10.1002/2015JA021894

Miyoshi Y, Fujiwara H, Jin H, Shinagawa H (2014) A global view of gravity waves in the thermosphere simulated by a general circulation model. J Geophys Res Sp Phys 119:5807-5820. https://doi.org/10.1002/2014JA019848

Miyoshi Y, Jin H, Fujiwara H, Shinagawa H (2018) Numerical study of traveling ionospheric disturbances generated by an upward propagating gravity wave. J Geophys Res Sp Phys 123:2141-2155. https://doi. org/10.1002/2017JA025110

Munro G (1950) Travelling disturbances in the ionosphere. Proc R Soc A Math Phys Eng Sci 202:208-223. https://doi.org/10.1098/rspa.1950.0095

Narayanan VL, Shiokawa K, Otsuka Y, Saito S (2014) Airglow observations of nighttime medium-scale traveling ionospheric disturbances from Yonaguni: statistical characteristics and low-latitude limit. J Geophys Res Sp Phys 119:9268-9282. https://doi.org/10.1002/2014JA020368

Nelson RA (1968) Response of the ionosphere to the passage of neutral atmospheric waves. J Atmos Terr Phys 30:825-835. https://doi. org/10.1016/S0021-9169(68)80034-0

Onishi T, Tsugawa T, Otsuka Y et al (2009) First simultaneous observations of daytime MSTIDs over North America using GPS-TEC and DEMETER satellite data. Geophys Res Lett 36:1-5. https://doi.org/10.1029/2009G L038156

Otsuka Y, Kotake N, Shiokawa K, et al (2011) statistical study of medium-scale traveling ionospheric disturbances observed with a GPS Receiver Network in Japan. In: Aeronomy of the Earth's Atmosphere and lonosphere. Springer Netherlands, Dordrecht, pp 291-299
Otsuka Y, Ogawa T, Saito A et al (2002) A new technique for mapping of total electron content using GPS network in Japan. Earth Planets Sp 54:63-70. https://doi.org/10.1186/BF03352422

Otsuka Y, Onoma F, Shiokawa K et al (2007) Simultaneous observations of nighttime medium-scale traveling ionospheric disturbances and $\mathrm{E}$ region field-aligned irregularities at midlatitude. J Geophys Res Sp Phys 112:1-9. https://doi.org/10.1029/2005JA011548

Otsuka Y, Shiokawa K, Ogawa T et al (2009) Spatial relationship of nighttime medium-scale traveling ionospheric disturbances and $\mathrm{F}$ region fieldaligned irregularities observed with two spaced all-sky airglow imagers and the middle and upper atmosphere radar. J Geophys Res Sp Phys 114:1-11. https://doi.org/10.1029/2008JA013902

Otsuka Y, Suzuki K, Nakagawa S et al (2013) GPS observations of medium-scale traveling ionospheric disturbances over Europe. Ann Geophys. https:// doi.org/10.5194/angeo-31-163-2013

Park J, Lühr H, Lee C et al (2014) A climatology of medium-scale gravity wave activity in the midlatitude/low-latitude daytime upper thermosphere as observed by CHAMP. J Geophys Res Sp Phys 119:2187-2196. https://doi. org/10.1002/2013JA019705

Pierce JA (1940) Mimno HR (1940) The reception of radio echoes from distant ionospheric irregularities. Phys Rev 57:95-105

Saito A, Fukao S, Miyazaki S (1998) High resolution mapping of TEC perturbations with the GSI GPS network over Japan. Geophys Res Lett 25:30793082. https://doi.org/10.1029/98GL52361

Saito S, Yamamoto M, Hashiguchi H (2008) Imaging observations of nighttime mid-latitude F-region field-aligned irregularities by an MU radar ultra-multi-channel system. Ann Geophys 26:2345-2352. https://doi. org/10.5194/angeo-26-2345-2008

Shinagawa H (2009) lonosphere simulation. J Natl Inst Inf Commun Technol 56:199-207

Shinagawa H, Miyoshi Y, Jin H, Fujiwara H (2017) Global distribution of neutral wind shear associated with sporadic E layers derived from GAIA. J Geophys Res Sp Phys 122:4450-4465. https://doi.org/10.1002/2016JA023778

Shiokawa K, Ihara C, Otsuka Y, Ogawa T (2003) Statistical study of nighttime medium-scale traveling ionospheric disturbances using midlatitude airglow imagers. J Geophys Res 108(A1):1052

Soicher H (1988) Traveling ionospheric disturbances (TIDs) at mid-latitudes: solar cycle phase dependence. Radio Sci 23:283-291. https://doi. org/10.1029/RS023i003p00283

Trinh QT, Ern M, Doornbos E et al (2018) Satellite observations of middle atmosphere-Thermosphere vertical coupling by gravity waves. Ann Geophys 36:425-444. https://doi.org/10.5194/angeo-36-425-2018

Tsugawa T, Kotake N, Otsuka Y, Saito A (2007) Medium-scale traveling ionospheric disturbances observed by GPS receiver network in Japan: a short review. GPS Solut 11:139-144. https://doi.org/10.1007/s1029 1-006-0045-5

Tsugawa T, Saito A, Otsuka Y, Yamamoto M (2003) Damping of large-scale traveling ionospheric disturbances detected with GPS networks during the geomagnetic storm. J Geophys Res Sp Phys 108:1-14. https://doi. org/10.1029/2002JA009433

Vadas SL, Liu HL (2009) Generation of large-scale gravity waves and neutral winds in the thermosphere from the dissipation of convectively generated gravity waves. J Geophys Res Sp Phys 114:1-25. https://doi. org/10.1029/2009JA014108

Vadas SL, Xu S, Yue J et al (2019) Characteristics of the quiet-time hot spot gravity waves observed by GOCE over the southern andes on 5 july 2010. J Geophys Res Sp Phys 124:7034-7061. https://doi.org/10.1029/2019J A026693

Zhou C, Zhao Z, Yang G et al (2012) Evidence of low-latitude daytime largescale traveling ionospheric disturbances observed by high-frequency multistatic backscatter sounding system during a geomagnetically quiet period. J Geophys Res Sp Phys 117:1-8. https://doi.org/10.1029/2012J A017605

\section{Publisher's Note}

Springer Nature remains neutral with regard to jurisdictional claims in published maps and institutional affiliations. 